\title{
Erratum to: Effect of seaweed extracts and plant growth regulators on high-frequency in vitro mass propagation of Lycopersicon esculentum L (tomato) through double cotyledonary nodal explant
}

\author{
S. Vinoth $\cdot$ P. Gurusaravanan $\cdot$ N. Jayabalan
}

Published online: 4 December 2011

(C) Springer Science+Business Media B.V. 2011

Erratum to: J Appl Phycol

DOI 10.1007/s10811-011-9717-9

Unfortunately, Figures 1 and 2 were interchanged when this article was produced and should appear a shown here.

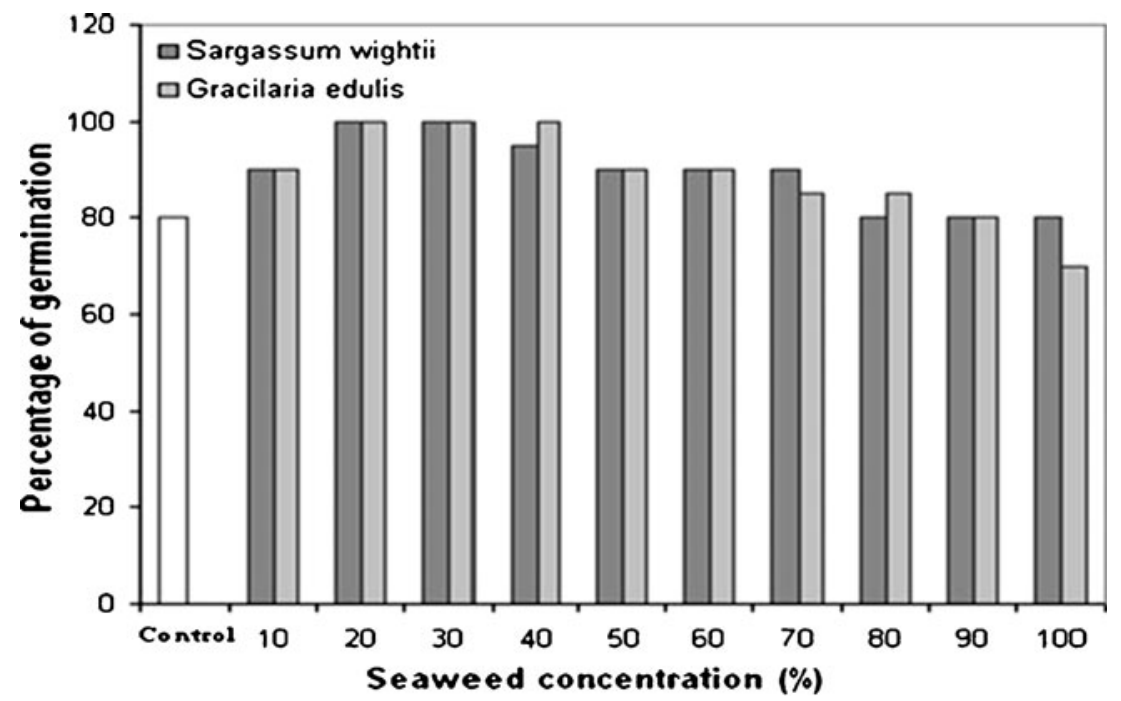

Fig. 1 Effect of MS basal medium, Gracilaria edulis and Sargassum wightii on in vitro seed germination of tomato. The medium was composed of MS salts and B5 vitamins, 3\% sucrose and seaweeds at different concentrations. MS medium without any seaweed extracts is used as a control

The online version of the original article can be found at http://dx.doi. org/10.1007/s10811-011-9717-9.

S. Vinoth $(\bowtie) \cdot$ P. Gurusaravanan $\cdot$ N. Jayabalan

Department of Plant Biotechnology, School of Life Sciences,

Bharathidasan University,

Tiruchirappalli-24,

Tamil Nadu, India

e-mail: vinogenes@gmail.com 
Fig. 2 Micropropagation of Lycopersicon esculentum L using double cotyledonary nodal explant. a and b Shoot bud regeneration on medium supplemented with $1.5 \mathrm{mg} \mathrm{L}^{-1}$ of TDZ and $1.5 \mathrm{mg} \mathrm{L}^{-1}$ of IBA after 20 days of subculture. c Shoot bud proliferation on MS medium. d Multiple shoot initiation and formation of mini shoots after four subcultures. e Mini shoots cultured on MS medium containing $30 \% \mathrm{G}$. edulis. f Shoot elongation and formation of basal callus at 3\% of sucrose on medium fortified with $1.2 \mathrm{mg} \mathrm{L}^{-1}$ of iP. g Rooting of in vitro regenerated shoots on MS medium supplemented with $50 \%$ of $S$. wightii. h Hardened in vitro-derived plant in the plant growth chamber and maintained in it for 7 days. i Acclimatized plant outside the plant growth chamber
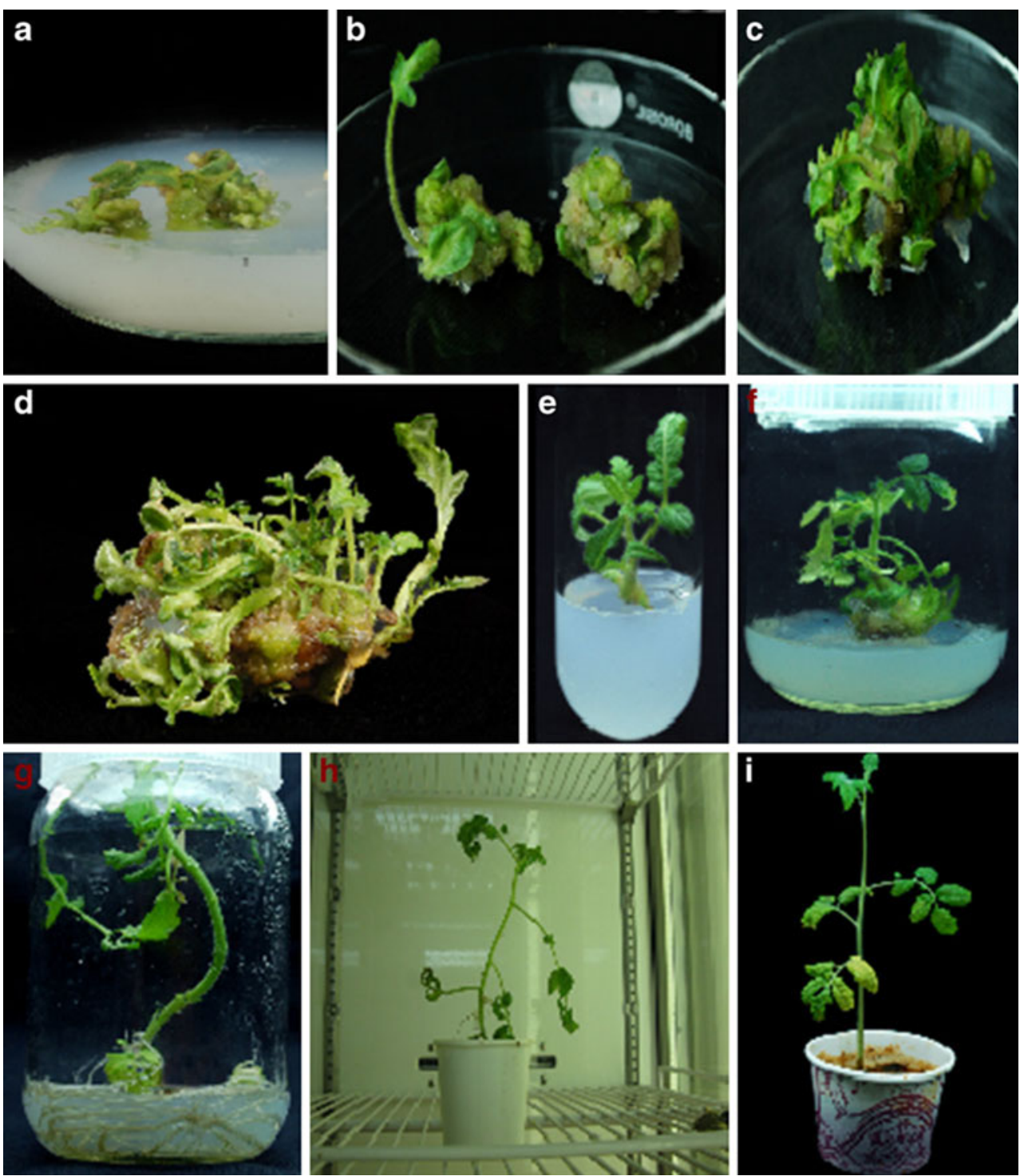